\title{
Noninvasive preimplantation genetic testing for aneuploidies (niPGT-A) and the principle of primum non nocere
}

\author{
Jose G. Franco Jr ${ }^{1}$, Felipe Dieamant ${ }^{1}$, Joao Batista A. Oliveira ${ }^{1}$ \\ ${ }^{1}$ Center for Human Reproduction Prof. Franco Jr., Ribeirão Preto, SP, Brazil
}

\begin{abstract}
Perhaps with the intention of obtaining larger amounts of free-DNA, some groups are routinely postponing and establishing free-DNA collection in culture medium for Noninvasive preimplantation genetic testing for aneuploidies (niPGT-A) to day 6 for all blastocysts. A meta-analysis served as the basis for such decision, since statistically similar live birth rates were observed when the transfers of euploid blastocysts were performed on day 5 versus day 6 However, the euploidy analysis was conducted in only two studies However, after including the two more studies we performed a new meta-analysis that clearly showed the risks of losing live births with the decision of adopting the 6 th day as the endpoint for gathering free-DNA. We would be losing $1.71 x$ more live births.
\end{abstract}

Keywords: Noninvasive preimplantation genetic test, niPGT-A, PGT-A

The development of preimplantation genetic tests for aneuploidies (PGT-A) has a history of over 25 years. Initially, the invasive method labeled 'preimplantation genetic screening' (PGS) received its first critiques when Mastenbroek et al. (2007) published a randomized controlled study in which PGS was indicated for advanced maternal age. Its use did not increase, but instead, significantly reduced the rates of ongoing pregnancies and live births (PGS $=24 \%$; Control=35\%). This was the first time in which the use of a PGT (PGS) did not fulfill the principle of primum non nocere. Thus, those who supported PGS started to attribute the burden of negative outcomes to imperfect FISH techniques in chromosome abnormality assessments and the fact that biopsies performed on the third day (embryo cleavage stage) would carry a high frequency of mosaicism. Hence, a new PGS method was developed, with the embryo biopsy on the 5th or 6th day of embryonic development and the use of new chromosome assessment techniques (comparative genomic hybridization-CGH; next-generation sequencing-NGS, etc.) that were safer in establishing chromosomal abnormalities, in addition to enabling the analysis of all 24 chromosomes.

Despite the substantial technological development in this area, doubts arose regarding the invasive approach (known as inPGT-A in recent years) due to the occurrence of a percentage of false-positive diagnoses in the blastocyst biopsy of the trophoblast, especially in situations of embryonic mosaicism. Greco et al. (2015) described the first births of healthy children with mosaicism after embryo transfer. This fact alerted experts in the area about the risk of discarding countless embryos with a potential for implantation. It is noteworthy that the percentage of blastocysts with mosaicism does not usually vary with age, although its frequency could reach up to $50 \%$ in some laboratories (Popovic et al., 2020). This would constitute the second time that a PGT-A (inPGT-A) was disrespecting the principle of primum non nocere.

$\mathrm{Xu}$ et al. (2016) described the birth of several children using noninvasive PGT-A (niPGT-A) through the col- lection of free-DNA, which was secreted into the embryonic culture medium during human embryo development from cleavage until the blastocyst stage. By using multiple annealing and looping-based amplification cycles (MALBAC) for whole-genome amplification (WGA), the authors performed NGS on the free-DNA obtained in the spent culture medium of the blastocysts on day $5(n=42)$ and were able to analyze all 24 chromosomes. In order to validate their results, they compared the chromosomes in the culture medium with their corresponding whole donated embryos. The authors found a significant correlation in the identification of chromosomal abnormalities (sensitivity: 0.882; specificity: 0.840). With this validated niPGT-A method, they performed chromosome screening on IVF embryos from seven couples with balanced translocation, azoospermia, and recurrent pregnancy loss. As a result, six of them achieved successful clinical pregnancies and healthy live births. This niPGT-A method avoids the need for embryo biopsy and, therefore, substantially increases the safety of its use. The approach has the potential for much wider chromosome screening applicability in clinical IVF on account of its optimal accuracy and noninvasiveness (Fang et al., 2019; Jiao et al., 2019; Rubio et al., 2019; Olcha et al., 2020).

Despite this promising start, difficulties in the use of niPGT-A have been reported, which could be solved. In principle, a validation program is mandatory for groups that are interested in conducting niPGT-A before collecting freeDNA in spent culture medium. In such training, the risk of contamination with the patient's granulosa cells should be discussed with embryologists, and the group should be taught the appropriate measures for denuding embryos to reduce the levels of free-DNA contamination submitted to niPGT-A to below $2 \%$. Currently, some softwares can already use artificial intelligence to detect cases suspected of contamination. In this situation, a new free-DNA collection is required. Also, the technique for collecting free-DNA must be strictly standardized for each laboratory. This includes special pipettes, culture plates suitable for reduced volumes of culture medium, and the determination of use of a sequential or continuous culture system, depending on each laboratory's routine. All groups submitted to the validation process need to be efficient in free-DNA collection protocols, and the results must be reliable in both fresh embryonic and frozen-thawed cycles.

Regarding chromosomal mosaicism, Vagnini et al. (2020) described an incidence rate of approximately $32 \%$ in human blastocysts, established by niPGT-A using the NGS platform and the cut-off adopted by specific software. However, it did not vary remarkably with age. Euploidy levels had a negative correlation with increasing age, whereas aneuploidy levels presented a positive correlation with it. Therefore, the careful interpretation of the mosaicism phenomenon, established by niPGT-A, should be a priority to avoid discarding potentially normal embryos. Unfortunately, these guidelines still require clarification. Even when adopting the precautions suggested above, it is important to remember that PGT techniques can select euploid em- 


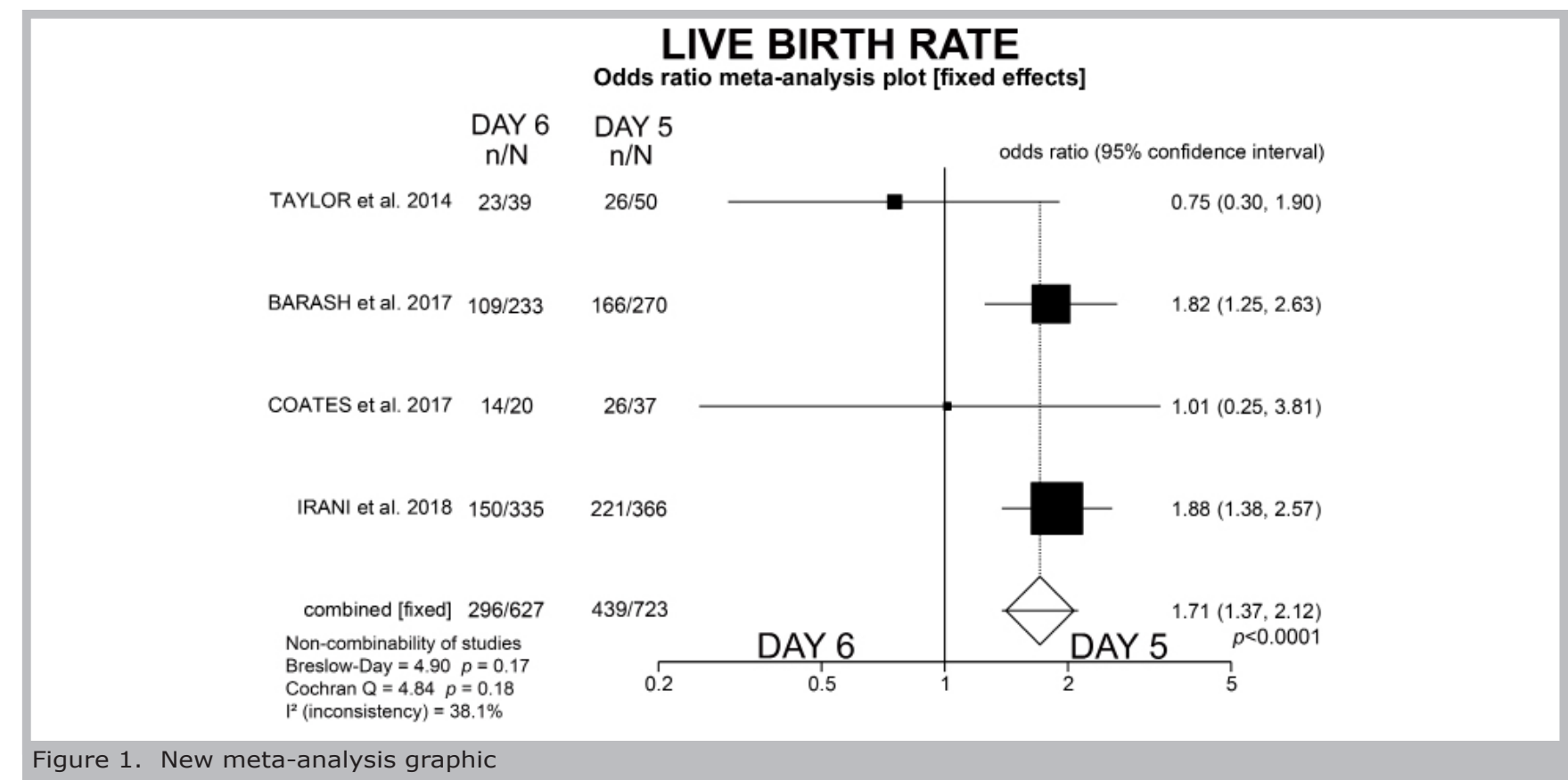

bryos within several embryos with euploidy, aneuploidy, and mosaicism. Nonetheless, euploids fail to implant in $30 \%$ to $40 \%$ of embryonic transfers. Therefore, the primary goal of laboratories should be to produce more euploid embryos.

On the other hand, perhaps with the intention of obtaining larger amounts of free-DNA, some groups are routinely postponing and establishing free-DNA collection in culture medium to day 6 for all blastocysts (Rubio et al., 2020). The meta-analysis published by Bourdon et al. (2019) served as the basis for such decision, since statistically similar live birth rates were observed when the transfers of euploid blastocysts were performed on day 5 versus day 6 (analysis subgroup). However, the euploidy analysis was conducted in only two studies (Barash et al., 2017; Coates et al., 2017). We highlight that the study by Taylor et al. (2014) and Irani et al. (2018) were not included in this meta-analysis subgroup regarding the transfer of euploid embryos (day 5 versus day 6 ).

Therefore, after including the data above, we performed a new meta-analysis that clearly showed the risks of losing live births with the decision of adopting the 6th day as the endpoint for gathering free-DNA. We would be losing $1.71 \mathrm{x}$ more live births, as shown in Figure 1. Collecting free-DNA on day 5 would be ideal, as long as its development and blastulation are compatible. Collection could also be performed on the 6th day since the embryo reaches blastulation later. However, even if it were euploid, it would produce an important reduction in the percentage of live births. Moreover, when Bourdon et al. (2019) evaluated both fresh and frozen-thawed cycles, without genetic analysis, they concluded that ART practitioners should preferably transfer D5 rather than D6 blastocysts.

In conclusion, establishing the 6 th day as a routine for collecting free-DNA for niPGT-A will undoubtedly be the third disobedience of PGT regarding the principle of primum non nocere.

\section{REFERENCES}

Barash OO, Ivani KA, Willman SP, Rosenbluth EM, Wachs DS, Hinckley MD, Pittenger Reid S, Weckstein LN. Association between growth dynamics, morphological parameters, the chromosomal status of the blastocysts, and clinical outcomes in IVF PGS cycles with single embryo transfer. J Assist Reprod Genet 2017;34:1007-6. PMID: 28560610 DOI: $10.1007 / \mathrm{s} 10815-017-0944-0$

Bourdon $M$, Pocate-Cheriet $K$, Finet de Bantel A, Grzegorczyk-Martin V, Amar Hoffet A, Arbo E, Poulain M, Santulli P. Day 5 versus Day 6 blastocyst transfers: a systematic review and meta-analysis of clinical outcomes. Hum Reprod. 2019; 34:1948-64. PMID: 31644803. DOI: 10.1093/humrep/dez163.

Coates A, Kung A, Mounts E, Hesla J, Bankowski B, Barbieri E, Ata B, Cohen J, Munné S. Optimal euploid embryo transfer strategy, fresh versus frozen, after preimplantation genetic screening with next generation sequencing: a randomized controlled trial. Fertil Steril 2017;107:723-30. PMID: 28139240. DOI: 10.1016/j.fertnstert.2016.12.022.

Fang $R$, Yang $W$, Zhao X, Xiong F, Guo C, Xiao J, Chen L, Song X, Wang H, Chen J, Xiao X, Yao B, Cai LY. Chromosome screening using culture medium of embryos fertilised in vitro: a pilot clinical study. J Transl Med. 2019;17:73. PMID: 30849973 DOI: 10.1186/s12967-019-1827-1.

Greco E, Minasi MG, Fiorentino F. Healthy Babies after Intrauterine Transfer of Mosaic Aneuploid Blastocysts. N Engl J Med. 2015;373:2089-90. PMID: 26581010 DOI: 10.1056/NEJMc1500421.

Jiao J, Shi B, Sagnelli M, Yang D, Yao Y, Li W, Sha L, Lu S, Li D, Wang $X$. Minimally invasive preimplantation genetic testing using blastocyst culture medium. Hum Reprod 2019;34:1369-79. PMID: 31251795 DOI: 10.1093/humrep/dez075

Irani M, O'Neill C, Palermo GD, Xu K, Zhang C, Qin X, Zhan Q, Clarke RN, Ye Z, Zaninovic N, Rosenwaks Z. Blastocyst development rate influences implantation and live birth rates of similarly graded euploid blastocysts. Fertil Steril. 2018;110:95-102.e1. PMID: 29908774. DOI: 10.1016/j. fertnstert.2018.03.032. 
Mastenbroek S, Twist $M$, van Echten-Arends J, Sikkema-Raddatz B, Korevaar JC, Verhoeve HR, Vogel NE, Arts EG, de Vris JW, Bossuyt PM, Buys CH, Heineman MJ, Repping $S$, van der Veen $F$. In vitro fertilization with preimplantation genetic screening. N Engl J Med 2007; 357:9-17. PMID: 17611204 . DOI: $10.1056 /$ NEJMoa067744.

Olcha M, Elzaky M, Jaremko M, John Z, Hensinger B, Lu $S$. A review of 1,504 autologous embryos evaluated using a non-invasive platform for preimplantation genetic testing for aneuploidy at a private clinic. Hum Reprod 2020;35:i373.

Popovic M, Dhaenens L, Boel A, Menten B, Heindryckx B. Chromosomal mosaicism in human blastocysts: the ultimate diagnostic dilemma. Hum Reprod Update. 2020; 26: 313-34. PMID: 32141501 DOI: 10.1093/humupd/dmz050.

Rubio C, Rienzi L, Navarro-Sánchez L, Cimadomo D, García-Pascual CM, Albricci L, Soscia D, Valbuena D, Capalbo A, Ubaldi F, Simón C. Embryonic cell-free DNA versus trophec- toderm biopsy for aneuploidy testing: concordance rate and clinical implications. Fertil Steril. 2019;112:510-9. PMID: 31200971 DOI: 10.1016/j.fertnstert.2019.04.038.
Rubio C, Navarro-Sánchez L, García-Pascual CM, Ocali O, Cimadomo D, Venier W, Barroso G, Kopcow L, Bahçeci M, Kulmann MIR, López L, De la Fuente E, Navarro R, Valbuena D, Sakkas D, Rienzi L, Simón C. Multicenter prospective study of concordance between embryonic cell-free DNA and trophectoderm biopsies from 1301 human blastocysts. Am J Obstet Gynecol. 2020:S0002-9378(20)30520-2.

PMID: 32470458. DOI: 10.1016/j.ajog.2020.04.035.

Taylor TH, Patrick JL, Gitlin SA, Wilson JM, Crain JL, Griffin DK. Comparison of aneuploidy, pregnancy and live birth rates between day 5 and day 6 blastocysts. Reprod Biomed Online. 2014;29:305-10. PMID: 25043891. DOI: 10.1016/j.rbmo.2014.06.001.

Vagnini LD, Petersen CG, Renzi A, Dieamant D, Oliveira JBA, Oliani AH, Canas MCT, Nakano R, Almodin CG, Marcondes C, Ceschin A, Amaral A, Soares JB, Lopes J, Franco AC, Franco Jr JG. Relationship between age and blastocyst chromosomal ploidy analysed by noninvasive preimplantation genetic testing for aneuploidies (niPGT-A). JBRA Assist Reprod. 2020. PMID: 32723707. DOI: 10.5935/15180557.20200061.

Xu J, Fang $R$, Chen L, Chen D, Xiao JP, Yang $W$, Wang $H$, Song X, Ma T, Bo S, Shi C, Ren J, L, Cai LY, Yao B, Xie XS, Lu $S$. Noninvasive chromosome screening of human embryos by genome sequencing of embryo culture medium for in vitro fertilization. Proc Natl Acad Sci USA. 2016; 113:1190712. PMID: 2768876 DOI: 10.1073/pnas.1613294113. 